(Aus dem physiologischen Laboratorium der Universität Bonn.)

\title{
Kann \\ bei vollkommener Entziehung der Nahrung der Glykogengehalt im Thierkörper zunehmen?
}

Von

W. Pnitiger.

Viele Forscher sind heute der allerdings nicht bewiesenen Ansicht, dass im thierischen Körper Kohlehydrate aus Stoffen entstehen können, die keine Kohlehydrate sind und auch solche nicht als Bestandtheile entbalten, was z. B. bei den Glykosiden der Fall ist. Es wäre demgemäss und besonders wegen der grossen Verbreitung der Glykoside denkbar, dass bei vollkommener Entziehung der Nahrung unter Umständen eine Vermehrung des Glykogengebaltes im Thierkörper sich vollziehen könnte.

Nun haben die Erfahrungen der Physiologen zu der berechtigten Schlussfolgerung geführt, dass bei Nahrungsmangel das thierische Leben durch den Verbrauch von Fett und Kohlehydraten vorzugsweise unterbalten wird. Dabei vermindert sich das Glykogen so schnell, dass schon nach wenigen Tagen nur noch sehr kleine Mengen dieser Substanz vorhanden sind, während das Fett viel länger vorhält. Ja man glaubt allgemein, dass bei fortgesetzter Nahrungsentziehung das Glykogen bei andauerndem Leben vollkommen aus allen Organen verschwinde.

Nachdem von mir vor Kurzem in einer eingehenden Untersuchung ${ }^{1}$ ) der Beweis geliefert worden ist, dass die bisher veröffentlichten Glykogenanalysen mit einem wechselnden, aber immer sehr grossen Beobachtungsfehler behaftet sind und viel zu kleine Werthe ergeben; nachdem ich besonders auf die Irrthümer aufmerksam gemacht habe, welche bei der Analyse von sogenannten Spuren des Glykogenes begangen worden sind, unterliegt es keinem Zweifel

1) E. Pflüger, Dieses Archiv Bd. 75 S. 120.

E. Pfläger, Archiv für Physiologie. Bd. 76. 
mehr, dass die Frage nach dem vollkommenen Schwinden des Glykogenes während des Hungerns einer erneuten Untersuchung bedarf. Ein unmittelbarer Beleg für diese Auffassung liegt in der von mir nachgewiesenen Thatsache ${ }^{\mathbf{1}}$ ), dass ein Hund nach 38 tägiger Nahrungsentziehung in $20 \mathrm{~g}$ seines Trockenfleisches (entsprechend etwa $100 \mathrm{~g}$ frischer Substanz) noch 0,0182 $\mathrm{g}$ Reinglykogen enthielt, das durch die Jodreaction und Invertirung als solches sicher gestellt ist. Es erscheint desshalb sehr wohl möglich. dass Leber und Muskeln niemals frei von Glykogen sind, so lange das Leben bestehr.

Wenn man bedenkt, wie stark der Stoffwechsel in den Tagen der ersten Hungerwoche das Glykogen verbraucht, so ist es sehr auffallend, dass sogar bei den Warmblütern nach länger als fünf Wochen dawernder Nahrungsentziehung noch immer Glykogen im Körper des Thieres vorhanden ist.

Aber noch viel auffallender erscheint die Beobachtung, welche ich $^{2}$ ) im März 1898 machte, dass winterschlafende Frösche, die aus dem Schlamme eines Tümpels herausgeholt und sofort der Untersuchung unterworfen worden waren, so grosse Massen von Glykogen enthielten, wie man sie kaum bei irgend einem in reichlichster Fütterung stehenden Säugethiere (Pferd ausgenommen) beobachten kann. Und diese Frösche hatten fast ein halbes Jahr keine Nahrung $\mathrm{zu}$ sich genommen.

Um Anhaltspunkte zur Erklärung der merkwürdigen Thatsache zu gewinnen, forderte ich den in meinem Laboratorium arbeitenden Herrn J. A th an asiu auf, die Veränderungen des Glykogengehaltes des Froschkörpers zu erforschen, welche durch die verschiedenen Jahreszeiten bedingt sind. Herr J. A thanasi u ${ }^{3}$ ) hat mit grossem Fleisse und stets an einer hinreichend grossen Zahl von Thieren die Untersuchung durchgeführt und die wichtigen Ergebnisse seiner Arbeit bereits veröffentlicht.

Die von dem genannten Forscher entworfene Curve, welche den Glykogengehalt des Thierkörpers als Function der Jahreszeiten darstellt, zeigt, dass im September das Maximum liegt, von dem ab eine so langsame Verminderung sich vollzieht, dass im März noch etwa $2 / 3$ des im Herbst aufgebäuften Vorrathes vorhanden sind. Dass

1) E. Pflüger, Dieses Archiv Bd. 75 S. 224, 225.

2) E. Pflüger, 'Dieses Arehiv Bd. 71 S. $318 \mathrm{ff}$.

3) Dieses Archiv Bd. 74 S. 561. 
der Stoffwechsel während des Winterschlafes nicht zum Stillstand kommt, geht auch noch in überzeugender Weise aus der von J. Athanasiu besonders betonten Thatsache hervor, dass die im October auf das Ueppigste entwickelten Fettkörper am Ende des Winterschlafes auf unscheinbare Reste geschrumpft sind. - Im Früh jahr sinkt der Glykogengehalt rasch auf ein Minimum und hebt sich während des Sommers nur sehr langsam, um im Herbst zum Maximum aufzusteigen.

Die von J. Athanasiu gefundene auffallende Thatsache, dass bei den Fröschen der Glykogengehalt des Körpers in der Jahreszeit, welche den Thieren die reichlichste Ernährung bietet, nämlich Frühjabr und Sommer, sehr viel geringer ist als im Winter, wo der Schlaf im Schlamme der Tümpel die Zufuhr der Nahrung für viele Monate ausschliesst; diese auffallende Thatsache bat der genannte Forscher gewiss richtig erklärt, wenn er darauf hinweist, dass der durch die hohe Temperatur des Sommers gesteigerte Stoffwechsel eine grössere Anhäufung des Glykogenes nicht zu Stande kommen lässt. Sobald mit dem Ende der warmen Jahreszeit die Temperatur sinkt, ohne dass die Nahrung abnimmt, tritt die Möglichkeit einer Ersparniss des Glykogenes ein, welches noch vor dem Beginn des Winterschlafes im September seinen höcbsten Stand erreicht, der den des Sommers um ein Mehrfaches übertrifft. Da nun nach J. A thanasiu mit dem Beginn des Winterschlafes, d. h. sobald die Zufuhr der Nahrung aufhört, der Glykogengehalt des Körpers zu wachsen aufhört und langsam aber stetig sinkt, fehlt es an einer Berechtigung, um eine fortwährende Neubildung von Glykogen im Körper der winterschlafenden Frösche anzunehmen. Weil der Vorrath des Glykogenes im Herbst so sehr den im Sommer übertrifft unci weil im Winter der Verbrauch des grossen Vorrathes sich so langsam vollzieht, erklärt sich die auf den ersten Blick räthselhafte Erscheinung, dass die Thiere zu der Zeit, wo sie am wenigsten fressen, das meiste Glykogen enthalten, und zu der Zeit, wo sie sich am reichlichsten ernähren, am ärmsten an dieser Substanz gefunden werden.

Der so langsame Verbrauch des Glykogenes im Winterschlaf ist natürlich in erster Linie durch die niedere Temperatur der Thiere und ihre Ruhe bedingt. Es scheint aber ausserdem, als ob das Fett in viel höherem Maasse als das Glykogen während. des Winterschlafes zur Bestreitung der Bedürfnisse des Stoffwechsels herangezogen würde. - 
Es ist von Wichtigkeit, daran zu erinnern, dass auch schon bei den winterschlafenden Säugethieren ganz ähnliche Verhältnisse die Aufmerksamkeit der Forscher auf sich gezogen haben.

Es sind hier besonders die Beobachtungen von Aeby ${ }^{1}$ ) und Voit ${ }^{2}$ ) zu erwähnen. Carl Voit, der den Glykogengehalt der Leber und Muskeln winterschlafender Murmelthiere untersuchte, sagt, dass diese Substanz hier in so grosser Menge ohne Zufuhr von Kohlehydraten auftritt, wie sonst nur bei reichlichster Nahrung. "Das Glykogen kann," so meint Voit, „in diesem Falle nur bei dem Zerfalle von Eiweiss oder von Fett sich abgespalten baben." $\mathrm{Eduard} \mathrm{Ku}_{\mathrm{l}} \mathrm{z}^{3}$ ) hat durch eine besondere Untersuchung die Angaben Voit's geprüft. Er zeigte, dass im Gegensatz zu Voit's Behauptung der Glykogengehalt winterschlafender Murmelthiere "weit zurückbleibt hinter den Mengen, die man bei reichlichster Füterung von Kaniuchen mit Kohlehydraten erhält." Nachdem E. Külz z) im December, Januar, Februar und März 4 winterschlafende Murmelthiere auf den Glykogengehalt der Leber untersucht hatte, findet er es "weit natürlicher, das Leberglykogen der Winterschläfer nicht als nengebildet, sondern als Rest von dem Glykogen aufzufassen, welches die Thiere beim Beginn des Winterschlafes haben". Luchsinger sprach sich schon 1875 in seiner Dissertation S. 19 in gleichem Sinne aus. „Die Thatsache des langsamen Verbrauches von Glyko„gen bei Winterfröschen findet ihr Analogon an dem Warmblüter „im Winterschlaf."

Die Untersuchungen von Luchsinger, E. Külz, J. A thanasiu sind also in voller Uebereinstimmung. Eine Neubildung von Glykogen während des Winterschlafes anzunehmen, liegt keine Berechtigung vor.

Claude Bernard, dem ein Theil der auf das Glykogen der Winterschläfer bezüglichen Thatsachen wohl bekannt war, meint, dass man den Winterschlaf und seine Beziehung zum Stoffwechsel nicht als einfache Nahrungsentziehung auffassen dürfe. Wie man darüber auch urtheilen möge, es bleibt wünschenswerth zu untersuchen, ob die Nahrungsentziehung unter sonst normalen Lebensbedingungen

1) Aeby, Ueber den Einfluss des Winterschlafes auf die Zusammensetzung der verschiedenen Organe des Thierkörpers.

2) Voit, Zeitschr. f. Biol. Bd. 14 S. 118.

3) E. Külz, Dieses Archiv Bd. 24 S. 76.

4) E. Külz, Dieses Archiv Bd. 24 S. 79. 
Kann bei vollkommener Entziehung der. Nahrung der Glykogengehalt etc. 5

zu einer Steigerung des Glykogengehaltes im Thierkörper zu führen vermöge.

Als ich mit der Literatur des Glykogenes mich beschäftigte und eingehendere Kenntniss von der grossen Untersuchung nahm, die Prof. Eduard Külz zur fünfzigjährigen Doctor-Jubelfeier des Leipziger Physiologen Carl Ludwig 1891 ausgeführt hat, wurde ich auf merkwürdige, hierher gehörige Ergebnisse aufmerksam. Die grosse Zahl der von Eduard $\mathrm{K} u ̈ l z$ ausgeführten Versuche gestattet eine statistische Verwerthung. Aus den Zahlen von Eduard Külz ergibt sich, dass die Nahrungsentziehung bei den Hühnern in den ersten 3 Tagen eine Periode der Abnahme des Glykogenes zur Folge hat, an welche sich eine Periode der Zunahme anschliesst. Külz selbst hat das in seinen Zahlen steckende Ergebniss nicht bemerkt. Ich werde also zuerst die Thatsachen mittheilen müssen, um die es sich hier handelt und demgemäss die grosse Tabelle VIII (S. 6 u. 7) aus der Festschrift mittheilen.

Betrachten wir zuerst die Leber allein, was schon darum nothwendig ist, weil bei vielen Versuchen der Glykogengehalt der Museulatur nicht bestimmt ist. Es handelt sich um nicht weniger als 30 an Hühnern ausgeführte Versucbe.

Als Grundlage für die rechnende Verwerthung der Tabelle VIII von Külz soll folgender Satz gelten. "Wie viel Glykogen "enthält auf die Einheit des Anfangsgewichts ein Huhn, "nachdem es kürzere Zeit, und wie viel, nachdem es „längere Zeit keine Nahrung erhalten hat?

$\mathrm{Zu}$ dem Ende theile ich die Versuche in 2 Gruppen. Gruppe I betrifft eine Hungerzeit von 2 bis 3 vollen Tagen, Gruppe II von 6 bis 10 vollen Tagen. - Die Tabelle, welche ich aus den einzelnen Zahlen von $\mathrm{K} \ddot{\mathrm{u}} \mathrm{z}$ berechnet habe, wird also leicht verständlich sein.

Tabelle I.

\begin{tabular}{c|c|c|c|c|c}
\hline \hline $\begin{array}{c}\text { Nummer } \\
\text { der } \\
\text { Gruppe }\end{array}$ & $\begin{array}{c}\text { Zahl der } \\
\text { Thiere } \\
\text { (Hühner) }\end{array}$ & $\begin{array}{c}\text { Mittleres } \\
\text { Anfangs- } \\
\text { gewicht in g }\end{array}$ & $\begin{array}{c}\text { Dauer der } \\
\text { Nahrungs- } \\
\text { entziehung in } \\
\text { Tagen }\end{array}$ & $\begin{array}{c}\text { Mittlerer Gehalt der Leber } \\
\text { an Glykogen }\end{array}$ \\
\hline I & 14 & 1553 & $2-3$ Tage & 0,059 & 0,038 \\
II & 18 & 1354 & $6-10$, & 0,074 & 0,055
\end{tabular}




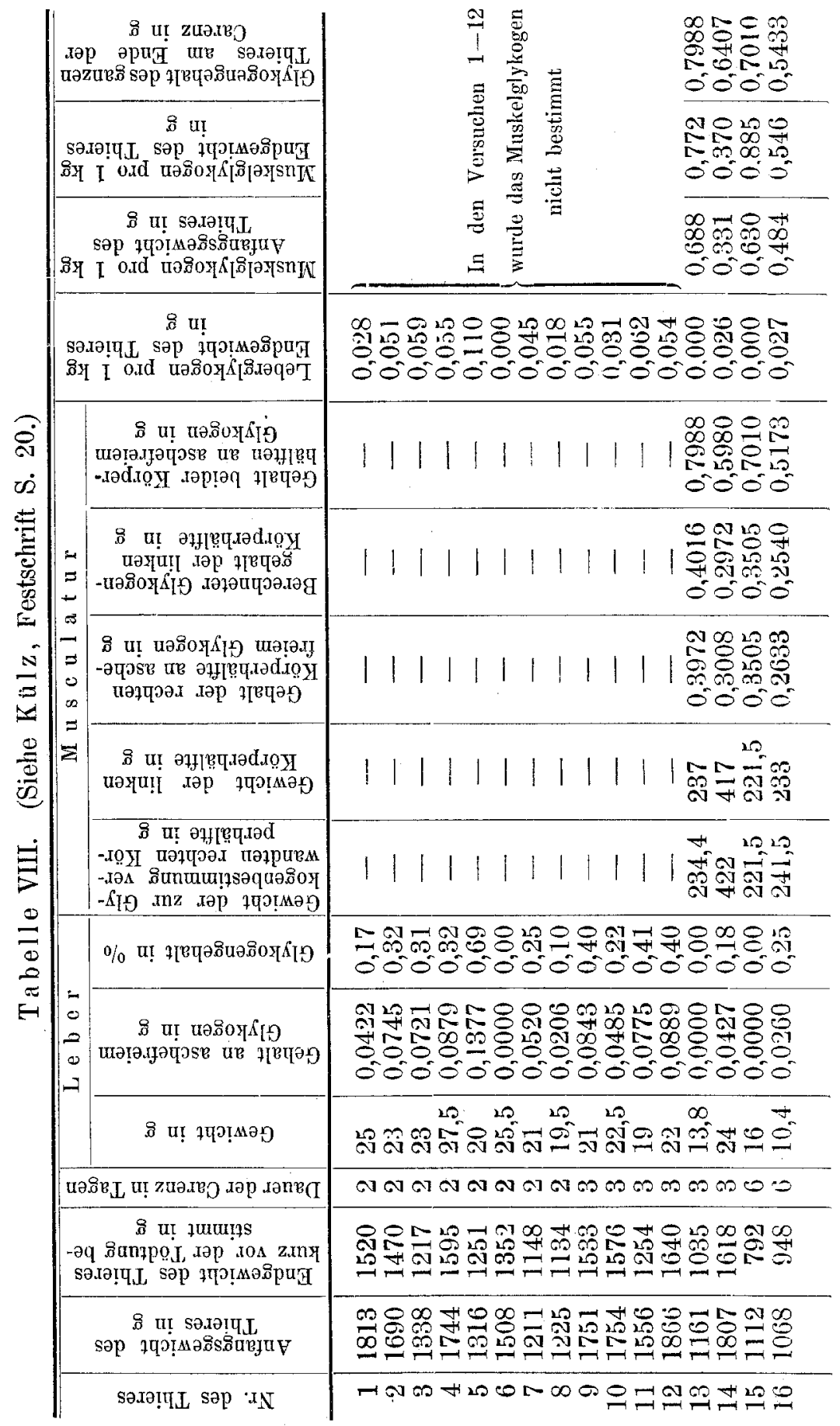


Kann bei vollkommener Entziehung der Nahrung der Glykogengehalt etc.

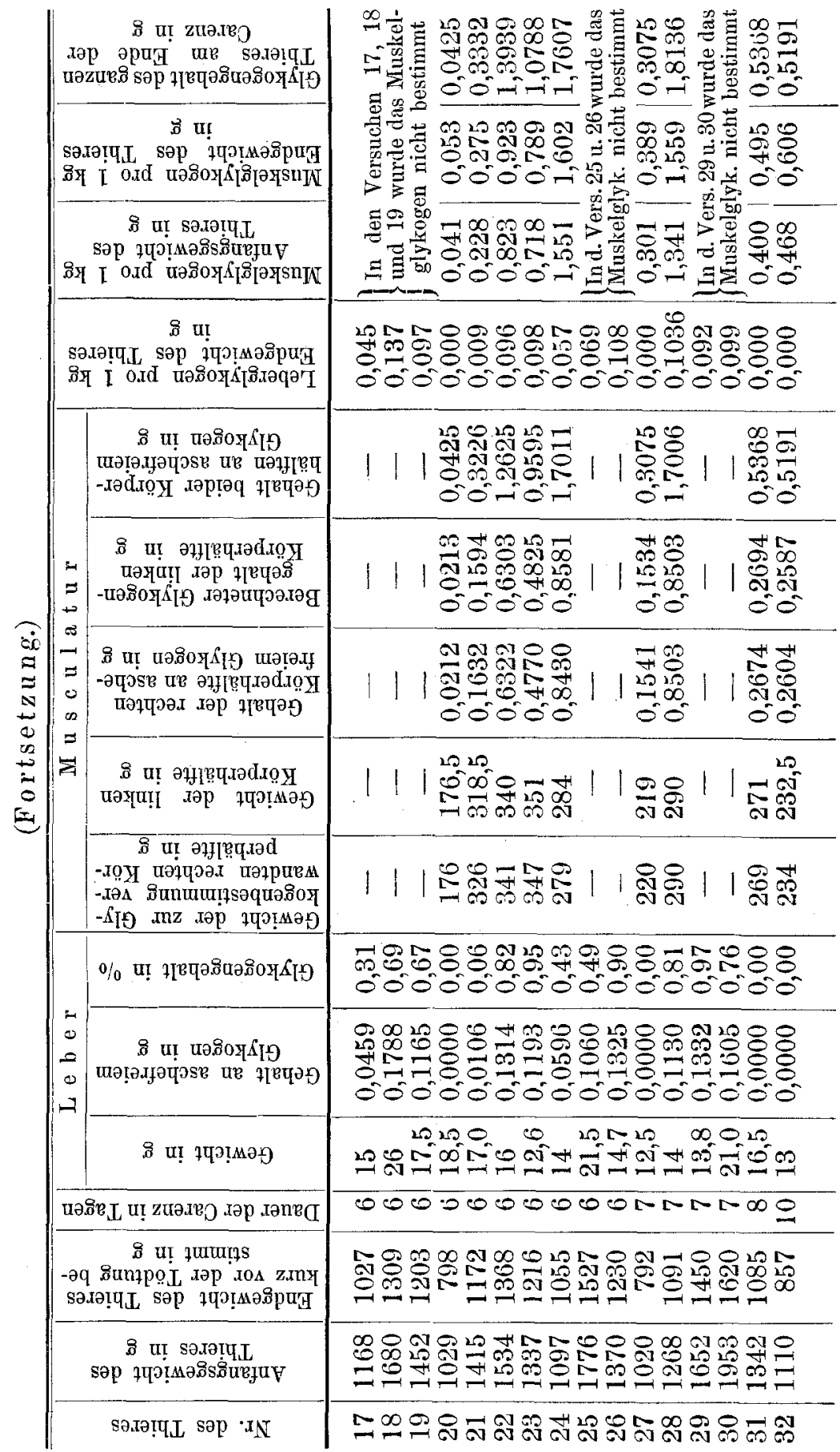


Das a $m$ 2. und 3. Hungertage in der Leber enthaltene Glykogen hat bei weiter fortgesetzter Nahruagsentziehung also um $44 \%$ zugenommen.

Es bleibt wegen der Deutung der Versuche die Thatsache zu besprechen, dass die Hühner, welche nach längerer Hungerzeit noch mehr Glykogen in der Leber hatten, im Allgemeinen leichtere Thiere waren. In Betracht käme die Rasse, weil es grosse und kleine Abarten gibt, ferner die Ernährung, weil sie das Gewicht erhöht, und endlich das Alter. Da die im Handel vorkommenden Hühner solche zu sein pflegen, welche keine Eier mehr legen, was auch mir jedesmal die Betrachtung der geschrumpften Eierstöcke bestätigt hat; so spielt das Alter hier keine wesentliche Rolle.

Um die Berechtigung dieses aus dem verschiedenen Gewicht der Thiere abgeleiteten Finwandes zu prüfen, theilen wir jede der beiden Gruppen wieder in je zwei Untergruppen, d. h. in die leichteren und schwereren Thiere, und untersuchen, $o b$ zwischen dem Gewicht und dem Glykogengehalt der Leber eine Beziehung auch bei solchen Hühnern sich herausstellt, die gleich lange Zeit gehungert haben. Ich entwerfe demgemäss aus der Tabelle VIII von $\mathrm{Külz}$ wieder zwei Tabellen.

Tahelle II (S. 9) bezeugt, dass das Körpergewicht, wenn überhaupt, einen nur sehr geringen Einfluss ausübt. Denn ein Unterschied des Gewichtes, der im Mittel $=1748-1292=456 \mathrm{~g}$ beträgt, bedingt auf die Gewichtseinheit von 1 Kilo Thier berechnet, nur einen Unterschied von 0,003 g Glykogen zu Gunsten der schwereren Thiere. In der Haupttabelle I handelt es sich aber nur um einen mittleren Gewichtsuntersehied von $200 \mathrm{~g}$, und was die Hauptsache, der grössere Glykogengehalt findet sich bei den im Mittel leichteren Thieren.

Da bei statistischen Untersuchungen die Zahl der Fälle möglichst zu steigern ist, müssen wir auch die Gruppe II, d. h. die Hühner, welche längere Zeit gehungert haben, in derselben Weise untersuchen, wie es für Gruppe I bereits geschehen ist. 
Kann bei vollkommener Entziehung der Nahrung der Glykogengehalt etc. 9

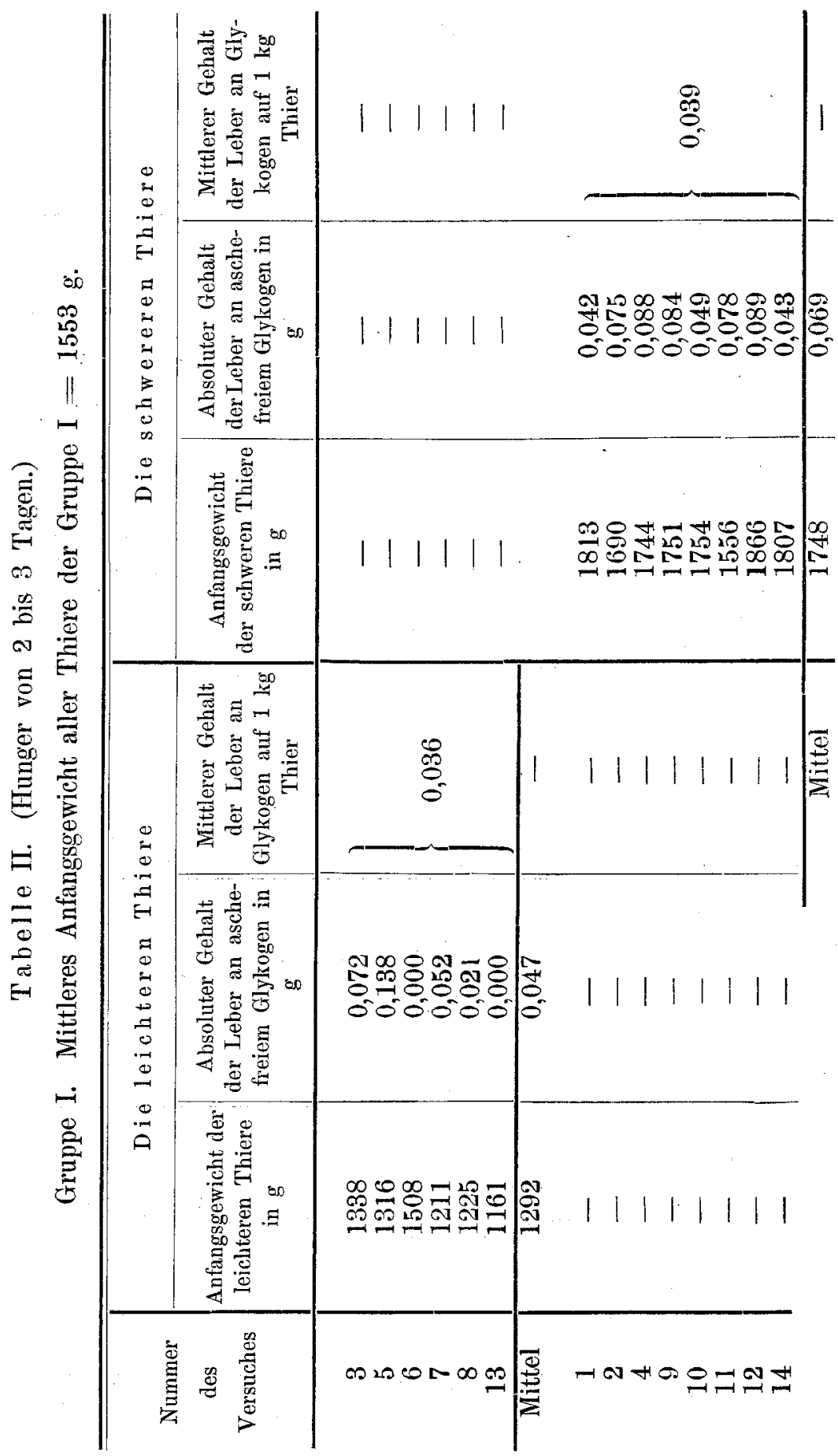


E. Pflüger:

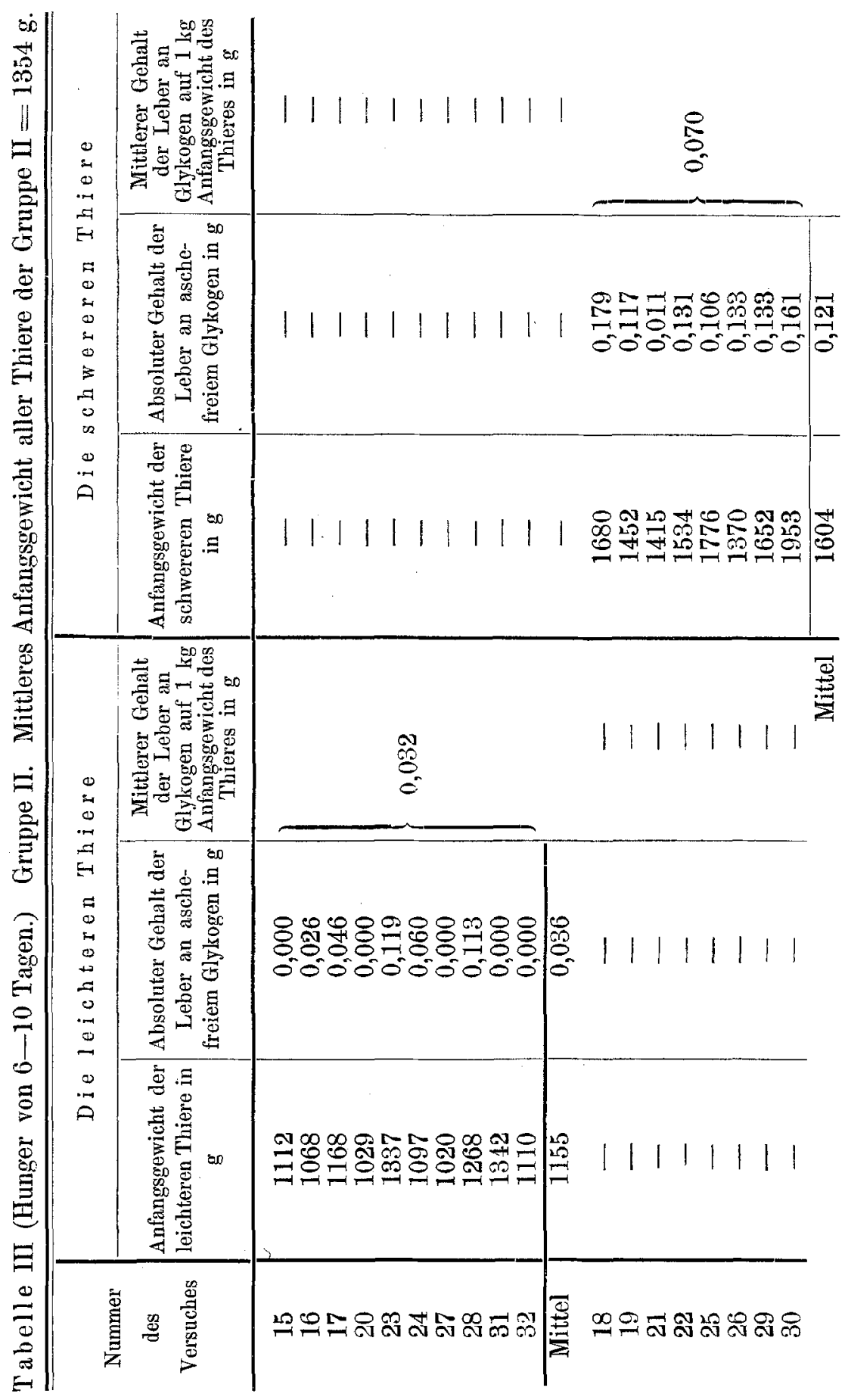


Der höchste Werth für den Glykogengehalt der Leber ist (Versuch 18) $0,179 \mathrm{~g}$ für ein Huhn von $1680 \mathrm{~g}$; also $0,107 \mathrm{~g}$ für ein Huhn von $1 \mathrm{~kg}$.

Also auch die Tabelle III bezeugt wie die Tabelle I und zwar viel entschiedener, dass der Körper der schwereren Thiere, auf die Einheit des Körpergewichtes bezogen, mehr Glykogen in der Leber enthält.

Wir dürfen deshalb jetzt mit Bestimmtheit voraussetzen, dass der höhere Glykogengehalt in der Leber der Hühner, welche länger gehungert hatten, seinen Grund nicht in dem verschiedenen mittleren Körpergewicht haben kann. Denn die Thiere, in deren Leber bei längerem Hungern mehr Glykogen geíunden worden ist, waren eben von geringerem Anfangsgewicht.

Da die Vorrathsstoffe, d. h. das Fett und die Kohlehydrate unter gewissen Bedingungen Wanderungen in thierischen Körper vollziehen, was durch N. Schulz und J. Athan asiu für das Fett in meinem Laboratorium durch viele quantitative Analysen über alle Zweifel erhoben worden ist, so erlangt die von mir soeben an das Licht gezogene Vermehrung des Leberglykogenes erst grundsätzliche Bedeutung, wenn die Veränderung des Glykogengehaltes nicht bloss für die Leber, sondern auch noch für den übrigen ganzen Körper des Thieres bestimmt wird. Das hat nun $\mathrm{Eduard} \mathrm{Külz}$ allerdings streng genommen nicht ausgefuhrt, sondern nur die Leber und die Gesammtmusculatur berücksichtigt. Da aber der Ablagerungsort für das Glykogen fast nur in der Leber und den Muskeln zu suchen ist und die Spuren, welche sich in anderen Organen finden, wenn es sich um Mengenverhältnisse handelt, nur wenig in Betracht kommen, so geben die Untersuchungen von $\mathrm{Eduard} \mathrm{Külz}$ doch wohl die nothwendige Unterlage zu einem Urtheile, ob die Gesamtmenge des Glykogenes in einer späteren Periode des Hungerns sich vermehrt.

Zur sicheren Beurtheilung der Thatsachen stelle ich die in Betracht kommenden Zahlen wieder aus Tabelle VIII von $K \ddot{u} l \mathrm{z}$ zusammen in folgender Tabelle IV (S. i2).

Das Ergebniss der in Tabelle $1 V$ aufgestellten Werthe ist also, dass das gesammte Glykogen, welches in $1 \mathrm{~kg} \mathrm{Huhn}$ nach 3 Hungertagen enthalten ist, bei weiter fortgesetzter Nahrungsentziehung von $0,485 \mathrm{~g}$ bis $0,678 \mathrm{~g}$ zugenommen hat, d. h. um 
Tabelle IV.

\begin{tabular}{|c|c|c|c|c|}
\hline \multirow{2}{*}{$\begin{array}{c}\text { Nummer der } \\
\text { Versuche }\end{array}$} & \multirow{2}{*}{$\begin{array}{l}\text { Anfangs- } \\
\text { gewicht des } \\
\text { Thieres in } \mathbf{g}\end{array}$} & \multirow{2}{*}{$\begin{array}{l}\text { Dauer der } \\
\text { Nahrungs- } \\
\text { entziehung }\end{array}$} & \multicolumn{2}{|c|}{$\begin{array}{c}\text { Gehalt der Leber und Musculatur des } \\
\text { Thieres an Glykogen }\end{array}$} \\
\hline & & & absolut in $\mathrm{g}$ & auf $1 \mathrm{~kg}$ Thier in $\mathrm{g}$ \\
\hline 13 & 1161 & 3 Tage & 0,799 & \multirow{3}{*}{0,485} \\
\hline 14 & 1807 & $3 n$ & 0,641 & \\
\hline Mittel & 1484 & & $0, \overline{720}$ & \\
\hline 15 & 1112 & $6 \mathrm{Tage}$ & 0,701 & \multirow{12}{*}{0,678} \\
\hline 16 & 1068 & $6 \quad$ & 0,543 & \\
\hline 20 & 1029 & & 0,043 & \\
\hline 21 & 1415 & & 0,333 & \\
\hline 22 & 1534 & & 1,394 & \\
\hline 23 & 1337 & & 1,079 & \\
\hline 24 . & 1097 & & 1,761 & \\
\hline 27 & 1020 & & 0,308 & \\
\hline 28 & 1268 & & 1,814 & \\
\hline 31 & 1342 & 8 & 0,537 & \\
\hline 32 & 1110 & 10 & 0,519 & \\
\hline Mittel & 1212 & & 0,821 & \\
\hline
\end{tabular}

Der höchste Werth für den absoluten Glykogengehalt von Leber und Musculatur ist (Versuch 28)

$$
\begin{aligned}
& 1,814 \mathrm{~g} \text { für ein Huhn von } 1268 \mathrm{~g}, \\
& \text { also } 1,437, " \quad " \quad, 1 \mathrm{~kg} \text {. }
\end{aligned}
$$

Ich gebe zu, die schwache Seite dieser Berechnung liegt in der zi kleinen Zahl der Versuche für die kürzere Zeit der Nahrungsentziehung. Denn es handelt sich um nur 2 Thiere.

Eine besondere Stütze erwächst aber der merkwürdigen Uebereinstimmung der beiden Versuchsreihen daraus, dass die auf die Leber bezüglichen Werthe zum sehr grossen Theil - es bandelt sich um 19 Versuche - an ganz anderen Thieren angestellt sind, als diejenigen, bei denen ausser dem Glykogen der Leber auch das der Muskeln bestimmt wurde.

Wenn die aus den Zahlen von $K \ddot{u} l z$ mit Nothwendigkeit abgeleiteten Ergebnisse der Wahrheit entsprechen, handelt es sich um Thatsachen, die aus mehr als einem Grunde von Bedeutung erscheinen. Ich entschloss mich desshalb, selber die Frage in die Hand zu nehmen.

Dabei kam es denn vor Allem darauf an, dass die Thiere, welche mit einander verglichen werden sollten, beim Beginne des Versuches so ähnlich waren, als es erreichbar ist. Ich wählte sie 
aus demselben Stalle, von derselben Art, möglichst demselben Gewicht und fütterte sie 8 Tage in demselben Stalle bei uns mit demselben Futter.

Das Gewicht der Thiere wurde bei Beginn der Nahrungsentziehung festgestellt, ebenso nach der Tödtung, die durch plötzliche Quetschung des Gehirns geschah. Dann wurden die Thiere schnell gerupft, die Federn gewogen und von dem Anfangsgewicht abgezogen. Die Federn machen annähernd ${ }^{1 / 10}$ des Thiergewichts aus.

$96,5 \mathrm{~g}$ Federn wurden in 1 Liter siedender Kalilauge von $4 \%$ in 1 Stunde gelöst und dann die Flüssigkeit auf Glykogen geprüft, was in sehr befriedigender Weise eben so gut wie bei der Leber oder dem Fleische ausführbar ist. Nach Zugiessen der vorschriftsmässigen Alkoholmenge zu dem Filtrat, welches nach der Brücke'schen Fällung erhalten worden war, schied sich bei tagelangem Stehen nichts aus.

Eduard Külz hat immer nur das halbe Huhn zur Untersuchung genommen, d. b. die Musculatur desselben. Ich nahm das ganze Thier und verfuhr wesentlich so, wie ich es bereits für die Bestimmung des gesammten Glykogenes beschrieben habe, das im Körper der Frösche enthalten ist ${ }^{1}$ ).

Das gerupfte Huhn wurde schnell seiner Eingeweide beraubt, die Steinchen aus dem Magen entfernt, die Muskeln abgeschnitten und alles mit Finschluss der Eingeweide zu einem feinen Brei zerhackt; zuletzt auch ebenso die Knochen mit Einschluss der Füsse und des Kopfes. Durch Kalilauge von $2 \%$ wird nach der von mir (s. dieses Archiv Bd. 75 S. 240) genauer beschriebenen Methode alles in Kalilauge Lösliche gewonnen, das Volum gemessen und ein bekannter Theil desselben anf Glykogen untersucht.

\section{Reihe I. A.}

Die beiden Thiere, welche 3 Tage hungern sollen, wiegen bei Anfang des Versuches: Huhn $1=1192 \mathrm{~g}$

$\begin{aligned} 2 & =1304 \mathrm{n} \\ \text { Summe } & =2496 \mathrm{~g} \\ \text { Federn } & =247 \mathrm{n} \\ \text { Hühner ohne Federn } & =2249 \mathrm{~g} \\ \text { Endgericht " } " & =2059,1 \mathrm{n} \\ \text { Die beiden Lebern } & =35,1 \mathrm{n}\end{aligned}$

1) Dieses Archiv Bd. 71 S. 319. 
Die beiden Thiere ohne Lebern werden in Brei verwandelt und in e in em Gefäss in Lösung gebracht, welche $3490 \mathrm{ccm}$ beträgt.

Von denselben werden $2 \mathrm{Mal} 200 \mathrm{ccm}$ entnommen. aschefreies Glykogen abgezogene Asche

Analyse $\alpha: 200 \mathrm{cem}=0,095 \mathrm{~g}$. . $0,003 \mathrm{~g}$

$\beta$ : verunglückt.

Also $=3490 \mathrm{cem}$ müssen enthalten: $1,658 \mathrm{~g}$ Glykogen.

Das aus den 2 Lebern erhaltene Glykogen beträgt:

$$
\begin{gathered}
\text { aschefrei abgezogene Asche } \\
0,044 \mathrm{~g} \quad \text { - } \quad 0,007 \mathrm{~g} .
\end{gathered}
$$

Das gesammte aschefreie Glykogen war also $=1,702 \mathrm{~g}$ entsprechend $2249 \mathrm{~g}$ Anfangsgewicht. Also

$1 \mathrm{~kg}$ Anfangsgewicht $=0,757 \mathrm{~g}$ Glykogen.

\section{Reihe I. B.}

Hunger von 6 Tagen.

Das eine der beiden Hühner starb schon am 4. Tage.

Das übrig Bleibende hatte mit Federn das Anfangsgewicht $=1176 \mathrm{~g}$ Die Federn betrugen . . . . . . . . . . . . $=96,5$, Also Anfangsgewicht ohne Federn . . . . . . . $=1079,5 \mathrm{~g}$ Das Endgewicht ohne Federn . . . . . . . . $=881,5$ "

Das Volum der alkalischen Lösung des ganzen Thieres mit Einschluss der Leber war $=1360 \mathrm{ccm}$.

Hiervon wurden entnommen $200 \mathrm{ccm}=158,8 \mathrm{~g}$. Thier.

$$
\text { aschefreies Glykogen abgezogene Asche }
$$

Analyse $\alpha: \quad 0,093 \mathrm{~g}$. . $0,006 \mathrm{~g}$

$$
" \beta: \frac{0,084, \quad . \quad . \quad 0,005,}{\text { Mittel } 0,0885}
$$

Demgemäss enthält $1 \mathrm{~kg}$ Huhn nach 6 tägigem Hunger noch $0,558 \mathrm{~g}$ Glykogen.

$$
\text { Also: }
$$

$1 \mathrm{~kg}$ Huhn nach 3 tägigem Hunger $=0,757 \mathrm{~g}$ Glykogen

$$
1 " \quad " 6 \text { " } " \quad \frac{=0,558 \%,}{=}
$$

Vom 3. bis 6. Hungertag hat das Glykogen abgenommen um $26,2 \%$. 


\section{Reihe II. A.}

Gruppe A. Hunger von 3 Tagen. Anfangsgewicht Endgewicht

$$
\begin{aligned}
& \left.\begin{array}{rl}
\text { Huhn } 5 & =1020 \mathrm{~g} \\
6 & =1276 \%
\end{array}\right\} 2079 \mathrm{~g} \\
& \text { Summe }=2296 \mathrm{~g} \\
& \text { Federn }=213 \% \quad \frac{=213 \%}{1866 \%}
\end{aligned}
$$

Huhn 5 und 6 in Kalilauge von $2 \%$ in Lösung gebracht. Erhalten $3655 \mathrm{ccm}$ Lösung. Zur Analyse $\alpha$ und $\beta$ entnommen je $400 \mathrm{ccm}$.

Analyse $\alpha$. Nach 1 maliger Reinigung des gewonnenen Glykogenes, dessen Lösung durch das Brücke'sche Reagens sich nicht trübte, schliesslich erhalten $1500 \mathrm{ccm}$ Flüssigkeit, die mit 2 Volumina Alkohol von 96 Vol. Procent gefällt wurden. Gefunden:

$$
\begin{aligned}
& 0,2568 \mathrm{~g} \text { Glykogen } \\
& 0,0105 \text { "Asche } \\
& \hline 0,2463 \mathrm{~g} \text { aschefreies Glykogen. }
\end{aligned}
$$

Folglich enthielten die 2 Hühner von $2083 \mathrm{~g}$ Anfangsgewicht 2,251 g aschefreies Glykogen

oder auf $1 \mathrm{~kg}$ Thier: $\quad 1,080 \mathrm{~g}$.

An aly se $\beta$. Genau wie Analyse $\alpha$ ausgefübrt. $400 \mathrm{ccm}$ Hühnerlösung lieferten $1250 \mathrm{ccm}$ eiweissfreie Glykogenlösung, welche enthielt:

$$
\begin{aligned}
& 0,2728 \mathrm{~g} \text { Glykogen } \\
& 0,0167 \mathrm{n} \text { Asche } \\
& \hline 0,256 \mathrm{l} \mathrm{g} \text { aschefreies Glykogen. }
\end{aligned}
$$

Demnach berechnet sich für 3655 cem kalische Hühnerlösung:

2,341 g aschefreies Glykogen.

Also enthält $1 \mathrm{~kg}$ Thier

$$
1,124 \mathrm{~g} \text { aschefreies Glykogen. }
$$

Demnach für $1 \mathrm{~kg}$ Thier nach

Analyse $\alpha: 1,080 \mathrm{~g}$ aschefreies Glykogen

$$
" \frac{\beta: 1,124 "}{\text { Mittel }=1,102 g \text { aschefreies Glykogen. }}
$$

$1 \mathrm{~kg} \mathrm{Huhn} \mathrm{enthält} \mathrm{nach} 3$ tägigem Hunger

$$
1,102 \mathrm{~g} \text { aschefreies Glykogen. }
$$




\section{Reihe II. B.}

\section{Hunger von 6 Tagen.}

Anfangsgewicht Endgewicht

$$
\begin{aligned}
& \text { Huhn } 7=1197 \mathrm{~g} \\
& \text { Huhn } 8=1064,5 \text {, } \\
& \text { Summe }=2261,5 \mathrm{~g} \quad 1920,5 \mathrm{~g} \\
& \text { Federn }=\frac{198,5 n}{2063,0 \mathrm{~g}} \cdot \cdot \frac{198,5 \mathrm{n}}{1722,0 \mathrm{~g} .}
\end{aligned}
$$

Die Analysen sind genau so wie bei A ausgeführt. Erhalten waren $3845 \mathrm{ccm}$ kalische Hühnerlösung. Angewandt zu jeder Analyse wurden $200 \mathrm{ccm}$. Erhalten in

$$
\begin{aligned}
& \text { aschefreies Glykogen abgezogene Asche } \\
& \text { Analyse } \alpha: \quad 0,096 \mathrm{~g} \text {. . . } 0,0155 \mathrm{~g} \\
& \text { " } \beta: \quad 0,099 " . .0,015 \% \\
& \text { " } \gamma: 0,096 " . .0,0135 " \\
& " \frac{\delta: \quad 9,096 . . \quad . \quad 0,0135 n}{\text { Mittel } 0,0967 \mathrm{~g} \text { aschefreies Glykogen. }}
\end{aligned}
$$

Die Gesammtmenge des Glykogenes ist demnach:

$$
1,859 \mathrm{~g} \text {. }
$$

$1 \mathrm{~kg} \mathrm{Huhn} \mathrm{enthält} \mathrm{nach} \mathrm{Hunger} \mathrm{von} 6$ Tagen

$$
0,901 \mathrm{~g} \text { Glykogen. }
$$

Das Ergebniss der Reihe II:

$1 \mathrm{~kg}$ Huhn nach Hunger von 3 Tagen $=1,102 \mathrm{~g}$ aschefr. Glykogen, $1 \mathrm{~kg} " \quad$ " " $\quad$ A $n=0,901 \%$ " $=0,201 \mathrm{~g}$ aschefr. Glykogen.

Oder vom 3. bis zum 6. Hungertag nahm das im Körper des Huhnes enthaltene Glykogen ab um

$$
18,2 \% \text {. }
$$

Bei allen hier mitgetheilten, von mir ausgeführten Analysen des Glykogenes ist die von mir $^{1}$ ) vorgeschlagene Correctur nicht ausgeführt, weil es sich in erster Linie nur um vergleichende Bestimmungen handelt.

Das Ergebniss beider Versuchsreihen besteht also darin, dass das Glykogen im Körper des hungernden Thieres fortwährend abnimmt, und zwar vom 3. bis zum 6 . Hungertage in recht beträchtlichem Maasse.

1) E. Pflüger, Dieses Archiv Bd. 75 S. 247. 
Ich gebe $\mathrm{zu}$, dass man meinen Versuchen die zu geringe Zahl der Fälle vorwerfen kann, die den Zufall nicht sicher ausschliessen. Da desshalb eine sehr grosse Zahl neuer Versuche ausgeführt werden müsste, so wäre zu prüfen, ob sich der grosse hierzu nöthige Aufwand an Arbeit und Mitteln rechtfertigen lässt. Ich glaube, dass dies nicht der Fall ist, wovon ich den Leser zu überzeugen gedenke.

Es fragt sich zuerst, ob keine Anhaltspunkte vorliegen, die es einigermaassen begreiflich machen, wie der Fehler in die von Eduard $\mathrm{K} u ̈ l \mathrm{z}$ ausgeführten Versuchsreihen gelangt ist.

Ich glaube zu wissen, wie sich das Räthsel löst. Ich habe oben bei den von $\mathrm{Külz}$ benutzten Thieren mit 3tägigem und 6tägigem Hunger die leichteren von den schwereren gesondert und auf diese Weise 4 Gruppen erhalten. In 3 dieser Gruppen war der mittlere Procentgehalt an Leberglykogen ziemlich gleich, gleichgültig ob die Thiere leicht oder schwer, 3 oder 6 Tage gehungert hatten. Aber eine 4. Gruppe unterschied sich von den 3 anderen ganz ausserordentlich, wie aus folgender Zusammenstellung erhellt:

\section{Leberglykogen auf $1 \mathrm{~kg}$ Thier}

Gruppe 1: Hunger von 2 bis 3 Tagen 0,036 leichtere Thiere,

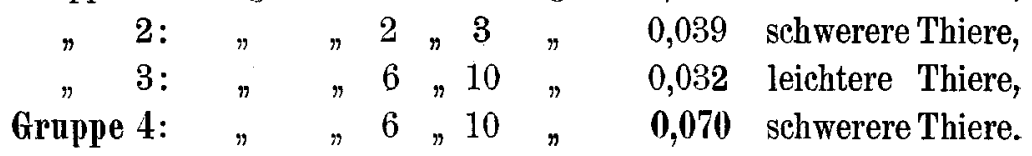

Da die Gruppe 4 aus 8 Thieren besteht, unter denen 7 durch sehr hohen Glykogengehalt ausgezeichnet sind, so genügt dies um der Gruppe $3+4$, d. h. den Thieren mit länger dauerndem Hunger das Uebergewicht $\mathbf{z u}$ verschaffen. Diese Gruppe schwererer Thiere hat $\mathrm{Edu}$ ard $\mathrm{Külz}$ vielleicht aus einer Quelle bezogen, zu einer Zeit, wo gerade die Versuche mit Hunger von 6-10 Tagen an der Reihe waren.

Das hohe Gewicht der Thiere der Gruppe 4, welches für eine gute Ernährung spricht, kann als wesentliche Ursache des ungewöhnlich hohen Glykogengehaltes nicht herangezogen werden, weil die Gruppe 2, welche ebenfalls aus 8 Thieren besteht, ein noch höheres mittleres Körpergewicht aufweist als Gruppe 4 und trotzdem viel weniger Glykogen enthält und nicht mehr als die Gruppen 1 und 3, welche leichteren Thieren entsprechen.

Es ist aber noch eine andere Möglichkeit vorhanden. Am Schlusse der Festschrift S. 53 findet sich folgende Bemerkung: 
"Gern hebe ich schliesslich dankend hervor die Zuverlässigkeit „und Ausdauer, mit der mich meine früheren und jetzigen Assistenten, „die Herren R. Külz, D. Sandmeyer und D. Blome bei dieser „Arbeit unterstützt haben."

Da man bei einer Glykogenanalyse keinen Assistenten braucht, kann ich diesen Ausspruch nicht anders verstehen, als dass 4 verschiedene Personen bei der Ausführung der Analysen betheiligt waren. Da für gewisse Hantirungen, von denen die Grösse der Beobachtungsfehler abhängt, bisher keine Vorschriften vorliegen, so sind Analysen, die von verschiedenen Personen ausgefürt wurden, unter einander nicht vergleichbar, wenn es sich wie hier um feinere Unterschiede handelt.

Wenn noch ein Zweifel bestehen bliebe, ob nicht doch in den Ergebnissen von E. $K \ddot{u} l_{\mathrm{z}}$ ein wahrer Kern entbalten sei, so würde er vollkommen beseitigt durch eine andere Versuchsreihe, welche derselbe Forscher an Tauben angestellt hat. Zur Erleichterung des Verständnisses theile ich zuerst die in Betracht kommende Tabelle $\mathrm{V}$ (S. 16) aus der Festschrift von Külz mit.

Tabelle V. (Siehe Külz, a. a. O. S. 16.)

\begin{tabular}{|c|c|c|c|c|c|c|}
\hline $\begin{array}{l}\text { Nr. } \\
\text { des } \\
\text { Ver- } \\
\text { suchs }\end{array}$ & $\begin{array}{l}\text { Anfangs- } \\
\text { gewicht } \\
\text { des } \\
\text { Thieres } \\
\text { in g }\end{array}$ & $\begin{array}{l}\text { End- } \\
\text { gewicht } \\
\text { des } \\
\text { Thieres } \\
\text { in g }\end{array}$ & $\begin{array}{c}\text { Wieviel } \\
\text { volle Tage } \\
\text { dauerte die } \\
\left.\text { Carenz? }{ }^{1}\right)\end{array}$ & $\begin{array}{c}\text { Gewicht } \\
\text { der Leber } \\
\text { in } g\end{array}$ & $\begin{array}{l}\text { Gehalt der } \\
\text { Leber an } \\
\text { aschefreiem } \\
\text { Glykogen } \\
\text { in } \mathrm{g}\end{array}$ & $\begin{array}{l}\text { Gehalt der ge- } \\
\text { sammten Mus- } \\
\text { culatur an } \\
\text { aschefreiem } \\
\text { Glykogen in } \mathrm{g}\end{array}$ \\
\hline 1. & 344 & 317 & 2 Tage & 3,7 & 0,0000 & - \\
\hline 2 & 362 & 327 & $2 "$ & 3,7 & 0,0000 & - \\
\hline 3 & 325 & 299 & $\overline{2}$ & 4,5 & 0,0093 & - \\
\hline 4 & 300 & 268 & & 3,7 & 0,0000 & - \\
\hline 5 & 333 & 298 & & 4,4 & 0,0509 & - \\
\hline 6 & 355 & 283 & & 3,5 & 0,0000 & 0,3386 \\
\hline 7 & 331 & 279 & 2 & 4,0 & 0,0000 & 0,3258 \\
\hline 8 & 346 & 315 & & 4,2 & 0,0041 & 0,4316 \\
\hline 9 & 297 & 258 & & 4,1 & 0,0000 & 0,4105 \\
\hline 10 & 365 & 295 & & 3,3 & 0,0000 & 0,1164 \\
\hline 11 & 339 & 251 & & 3,0 & 0,0000 & 0,2897 \\
\hline 12 & 349 & 256 & 5 & 3,0 & 0,0000 & 0,4934 \\
\hline 13 & 344 & 258 & 6 & 3,4 & 0,0000 & 0,2785 \\
\hline 14 & 392 & 267 & 7 & 4,2 & 0,0000 & 0,1723 \\
\hline 15 & 348 & 256 & 7 & 4,0 & 0,0000 & 0,1570 \\
\hline 16 & 365 & 277 & 7 & 3,0 & 0,0000 & 0,1834 \\
\hline 17 & 380 & 263 & & 3,0 & 0,0000 & 0,2451 \\
\hline
\end{tabular}

1) Sämmtliche Thiere wurden erst, nachdem der hropf leer geworden, auf Carenz gesetzt. 
Aus Tabelle $\mathrm{V}$ von $\mathrm{K} \ddot{u} \mathrm{lz}$ leite ich nun 2 neue Tabellen $\mathrm{ab}$, die ich mit $A$ und $B$ bezeichne. In Tabelle $A$ will ich den mittleren Glykogengehalt der Leber feststellen, je nachdem die Tauben kürzere ( 2 bis 4 Tage) oder längere Zeit ( 5 bis 8 Tage) gehungert haben.

Tabelle A.

\begin{tabular}{c|c|c|c|c}
\hline $\begin{array}{c}\text { Zahl der } \\
\text { Thiere }\end{array}$ & $\begin{array}{c}\text { Mittleres An- } \\
\text { fangsgewicht } \\
\text { der Tauben } \\
\text { in g }\end{array}$ & $\begin{array}{c}\text { Dauer der } \\
\text { Nahrungs- } \\
\text { entziehung } \\
\text { in Tagen }\end{array}$ & $\begin{array}{c}\text { Mittlerer Gehalt } \\
\text { der Leber an } \\
\text { aschefreiem Gly- } \\
\text { kogen in g }\end{array}$ & $\begin{array}{c}\text { Mittlerer Gehalt der } \\
\text { Leber auf 1 kg An- } \\
\text { fangsgewicht der } \\
\text { Tauben in g }\end{array}$ \\
\hline \multirow{3}{*}{11} & 336 & $2-4$ & 0,006 & 0,018 \\
6 & 363 & $5-8$ & 0,000 & 0,000
\end{tabular}

Dieser Versuch bezeugt, dass der längeren Hungerzeit der grössere Glykogenverlust entspricht.

Tabelle B soll ausser dem Glykogen der Leber auch das der gesammten Musculatur in gleicher Richtung wie bei Tabelle A darstellen.

Tabelle B.

\begin{tabular}{|c|c|c|c|c|c|}
\hline $\begin{array}{c}\text { Zahl } \\
\text { der } \\
\text { Thiere }\end{array}$ & $\begin{array}{l}\text { Mittleres } \\
\text { Anfangs- } \\
\text { gewicht } \\
\text { der Tauben }\end{array}$ & $\begin{array}{l}\text { Dauer der } \\
\text { Nahrungs- } \\
\text { entziehung } \\
\text { in Tagen }\end{array}$ & $\begin{array}{l}\text { Mittlerer Ge- } \\
\text { halt der Leber } \\
\text { und Muskeln } \\
\text { an asche- } \\
\text { freiem Gly- } \\
\text { kogen in } \mathrm{g}\end{array}$ & $\begin{array}{l}\text { Mittlerer Gehalt } \\
\text { der Leber und } \\
\text { Muskeln an } \\
\text { aschefreiem Gly- } \\
\text { kogen auf } 1 \mathrm{~kg} \\
\text { Anfangsgewicht } \\
\text { in } \mathrm{g}\end{array}$ & $\begin{array}{c}\text { Besondere Be- } \\
\text { merkungen }\end{array}$ \\
\hline 6 & 338 & $3-4$ & 0,327 & 0,967 & $\begin{array}{l}\text { Es sind hier ver- } \\
\text { werthet: Ver- } \\
\text { such } 6,7,8,9 \text {, } \\
10,11 \text { (Siehe } \\
\text { K ülz, a. a. } 0 . \\
\text { S. 16) }\end{array}$ \\
\hline 6 & 363 & $5-8$ & 0,260 & 0,716 & $\begin{array}{l}\text { Es sind die Ver- } \\
\text { suche } 12,13, \\
14,15,16,17 \\
\text { von } \mathrm{Kül} \text { z (a. a. } \\
\text { O. S. 16) }\end{array}$ \\
\hline
\end{tabular}

Bei den hungernden Thieren betrug der höchste Glykogengehalt der Leber (Versuch 5) $0,051 \mathrm{~g}$; also für

$1 \mathrm{~kg}$ Anfangsgewicht $=0,153$ Leberglykogen; der höchste Glykogengehalt der gesammten Musculatur (Versuch 12) war $0,4934 \mathrm{~g}$ oder

$1 \mathrm{~kg}$ Anfangsgewicht $=1,4 \mathrm{~g}$. 
Der grösste Werth für das Glykogen der Muskeln findet sich also bei der Gruppe der längere Zeit, für die Leber bei den kürzere Zeit hungernden Thieren.

Der kleinste Werth für den Glykogengehalt der Muskeln findet sich bei den kürzere Zeit hungernden Thieren (Versuch 10) mit $0,1164 \mathrm{~g}$; für den Glykogengehalt der Leber bei den längere Zeit hungernden Thieren.

Es schwankt also bei den Hungerthieren der Glykogengehalt der Leber von 0,000 his 0,051 für 1 Thier; der Glykogengehalt der Muskeln von 0,1164 bis $0,4934 \mathrm{~g}$ für 1 Thier.

Diese Versuche bezeugen aber im Mittel, dass das Glykogen bei fortgesetzter Nahrungsentziehung sowohl in der Leber als in den Muskeln stetig abnimmt.

Wenn man also die Untersuchungen in Betracht zieht, welche $\mathrm{Edu}$ ard $\mathrm{Kül} \mathrm{z}$ an winterseblafenden Murmelthieren und J. A than asiu an winterschlafenden Fröschen angestellt haben, um die Veränderungen des Glykogengehaltes im Thierkörper zu bestimmen; wenn man ferner die von mir hier mitgetheilten Thatsachen nur als eine Bestätigung der Ergebnisse der beiden Forscher auffassen kann, so folgt, dass das Glykogen bei dauernder Nahrungsentziehung stetig abnimmt. Es ist also keine Berechtigung vorhanden, mit Carl Voit anzunehmen, dass bei Nahrungsentziehung oder im Winterschlaf Glykogen, wie dieser Forscher sich ausdrückt, durch Abspaltung von Fett oder Eiweiss entstehe. 